\title{
Body iron stores and risk of type 2 diabetes: results from the European Prospective Investigation into Cancer and Nutrition (EPIC)-Potsdam study
}

\author{
J. Montonen • H. Boeing • A. Steffen • R. Lehmann • \\ A. Fritsche • H.-G. Joost • M. B. Schulze • T. Pischon
}

Received: 23 February 2012 / Accepted: 6 June 2012 / Published online: 1 July 2012

(C) The Author(s) 2012. This article is published with open access at Springerlink.com

\begin{abstract}
Aims/hypothesis The aim of this study was to prospectively examine the association between body iron stores and risk of type 2 diabetes.

Methods We designed a case-cohort study among 27,548 individuals within the population-based European Prospective Investigation into Cancer and Nutrition (EPIC)-Potsdam study. During 7 years of follow-up, 849 incident cases of type 2 diabetes were identified. Of these, 607 remained for analyses after exclusion of participants with missing data or abnormal glucose levels at baseline. A sub-cohort of 2,500 individuals was randomly selected from the full cohort, comprising 1,969 individuals after applying the same exclusion criteria.

Results After adjustment for age, sex, BMI, waist circumference, sports activity, bicycling, education, occupational activity, smoking habit, alcohol consumption and circulating levels of $\gamma$-glutamyltransferase, alanine aminotransferase, fetuin-A, high-sensitivity C-reactive protein, adiponectin,
\end{abstract}

J. Montonen $\cdot$ H. Boeing $\cdot$ A. Steffen $(\bowtie) \cdot$ T. Pischon

Department of Epidemiology,

German Institute of Human Nutrition Potsdam-Rehbruecke,

Arthur-Scheunert-Allee 114-116,

14558 Nuthetal, Germany

e-mail: annika.steffen@dife.de

R. Lehmann

Central Laboratory/Clinical Chemistry and Pathobiochemistry,

University Hospital Tübingen,

Tübingen, Germany

R. Lehmann · A. Fritsche

Paul Langerhans Institute Tübingen (Inst. for Diabetes Research

and Metabolic Diseases of the Helmholtz Centre Munich

at the University of Tübingen),

Tübingen, Germany

A. Fritsche

Department of Internal Medicine IV, University of Tübingen,

Tübingen, Germany
HDL-cholesterol and triacylglycerol, higher serum ferritin concentrations were associated with a higher risk of type 2 diabetes (RR in the highest vs lowest quintile, $1.73 ; 95 \% \mathrm{CI}$ $1.15,2.61 ; p_{\text {trend }}=0.002$ ). No significant association was observed for soluble transferrin receptor (RR 1.33; 95\% CI $\left.0.85,2.09 ; p_{\text {trend }}=0.50\right)$. The soluble transferrin receptor-toferritin ratio was significantly inversely related to risk (RR $0.61 ; 95 \%$ CI $0.41,0.91 ; p_{\text {trend }}=0.02$ ).

Conclusions/interpretation High ferritin levels are associated with higher risk of type 2 diabetes independently of established diabetes risk factors and a range of diabetes biomarkers whereas soluble transferrin receptor concentrations are not related to risk. These results support the hypothesis that higher iron stores below the level of haemochromatosis are associated with risk of type 2 diabetes.

Keywords Body iron store · Ferritin · Soluble transferrin receptor · Type 2 diabetes

\section{H.-G. Joost}

Department of Pharmacology,

German Institute of Human Nutrition Potsdam-Rehbruecke, Nuthetal, Germany

M. B. Schulze

Department of Molecular Epidemiology, German Institute of Human Nutrition Potsdam-Rehbruecke, Nuthetal, Germany

T. Pischon

Molecular Epidemiology Group,

Max Delbrück Center for Molecular Medicine (MDC),

Berlin-Buch, Germany 


$\begin{array}{ll}\text { Abbreviations } \\ \text { ALT } & \text { Alanine aminotransferase } \\ \text { EPIC } & \begin{array}{l}\text { European Prospective Investigation into } \\ \text { Cancer and Nutrition }\end{array} \\ \text { GGT } & \gamma \text {-Glutamyltransferase } \\ \text { hs-CRP } & \text { High-sensitivity C-reactive protein } \\ \text { sTfR } & \text { Soluble transferrin receptor } \\ \text { TfR } & \text { Transferrin receptor }\end{array}$

\section{Introduction}

Impaired glucose metabolism and diabetes mellitus are common clinical manifestations of iron overload in patients with haemochromatosis [1], a disorder of abnormal iron absorption resulting in the progressive accumulation of iron in inner organs. The precise molecular mechanisms underlying the pathogenesis of iron-overload-related diabetes have not been identified but the initial glucose abnormalities include insulin resistance and hyperinsulinaemia, followed by impaired insulin secretion [2]. Elevated iron stores below the levels of known iron overload syndromes have also been implicated in the aetiology of diabetes [2, 3]. However, so far only a few studies have prospectively examined the association between body iron stores and risk of diabetes [4-7].

Serum ferritin concentration is by far the most commonly used indicator of body iron stores in epidemiological studies. The use of serum ferritin in assessing iron stores, however, is complicated because ferritin is also an acute-phase protein that may be elevated in inflammation, liver disease and cancer [8]. Sub-clinical inflammation, which is usually observed in individuals with obesity and the metabolic syndrome, is associated with increased risk of type 2 diabetes [9]. It is thus unclear whether the association of ferritin with type 2 diabetes reflects these other abnormalities. Circulating ferritin levels have also been shown to be positively associated with circulating level of hepatic enzymes [4, 10] and markers of dyslipidaemia [11, 12], and negatively associated with adiponectin levels [4]. Thus, in a recent nested case-control study the association observed between serum ferritin and diabetes risk disappeared after adjustment for components of the metabolic syndrome [13].

Furthermore, serum ferritin concentrations may not be an accurate reflection of the intracellular iron pool, which is likely to be responsible for iron-related oxidative stress [14]. Serum soluble transferrin receptor (sTfR) concentration has been suggested as a more accurate measure of available body iron $[15,16]$. The level of circulating sTfR reflects the availability of iron in the body and it can be used in the clinical setting to distinguish between iron-deficiency anaemia and anaemia due to chronic diseases [16]. When the iron-transferrin complex binds to its cellular receptor (TfR), iron enters cells and the soluble extracellular domain of TfR is liberated into the circulation [17]. Serum levels of this soluble form (sTfR) are therefore directly proportional to tissue TfR concentration. Circulating sTfR levels correlate inversely with body iron stores and thus reflect, inversely, intracellular iron storage, and the ratio of sTfR to ferritin has been proposed as a preferred measure of body iron stores to quantifiably reflect body iron over the entire spectrum of iron balance $[18,19]$.

The objective of this study was to examine prospectively the association of body iron stores, as assessed by circulating ferritin concentrations and sTfR, as well as by the ratio of sTfR to ferritin, with risk of incident type 2 diabetes in a population-based cohort. We also investigated whether this association is independent of inflammation as well as markers of different metabolic pathways that may be involved in the pathogenesis of type 2 diabetes.

\section{Methods}

Study population The EPIC-Potsdam study is part of the multicentre European Prospective Investigation into Cancer and Nutrition (EPIC) [20, 21]. In Potsdam, Germany, 27,548 individuals $(16,644$ women mainly aged $35-65$ years and 10,904 men mainly aged 40-65 years) were recruited from the general population between 1994 and 1998 [22]. The baseline examination included anthropometric measurements, a personal health interview, a health questionnaire and blood sampling. Informed consent was obtained from participants; approval was given by the Ethics Committee of the Medical Association of the State of Brandenburg, Germany.

The health interview and the questionnaire elicited information on educational attainment, smoking and alcohol consumption habits, and occupational and leisure-time physical activity. The latter was assessed as sports activity and cycling, both calculated as the average time spent per week during the 12 months before recruitment. Waist circumference was measured midway between the lower rib margin and the superior anterior iliac spine to the nearest $0.5 \mathrm{~cm}$ with a non-stretching tape applied horizontally [23] Weight and height were measured without shoes in light clothing and BMI was calculated as $\mathrm{kg} / \mathrm{m}^{2}$.

The prevalence of diabetes at baseline was evaluated by a physician using information on self-reported medical diagnoses, medication records and ongoing nutritional therapy. Uncertainties regarding a proper diagnosis were clarified with the participant or their physician.

Incident cases of diabetes were identified during followup until 31 August 2005 via self-reports of a diabetes 
diagnosis, diabetes-relevant medication or dietary treatment related to diabetes. All incident cases were verified by questionnaires mailed to the diagnosing physician, requesting details of the date and type of diagnosis, diagnostic tests and treatment of diabetes. Only individuals with a diagnosis of type 2 diabetes that was confirmed by a physician (ICD10; www.who.int/classifications/icd/en/) and a diagnosis date after the baseline examination were considered as incident cases of type 2 diabetes.

Our study was conducted in a prospective case-cohort design [24]. For this, we selected a sub-cohort of 2,500 individuals from all participants in the EPIC-Potsdam study who provided blood samples at baseline $(n=26,444)$. Of these, 1,969 participants remained for analyses after excluding participants with any history of diabetes at baseline or with self-reported but unverified diabetes during follow-up, with missing values for study variables or with abnormal plasma glucose (random glucose $11.1 \mathrm{mmol} / 1$ or more, or fasting glucose $7.0 \mathrm{mmol} / 1$ or more, or less than $2.8 \mathrm{mmol} / \mathrm{l}$ ). By randomly selecting a sub-cohort and using appropriate statistics for this research design, the results are expected to be generalisable to the entire cohort without the need of biomarker measurements in the entire EPIC-Potsdam study. During an average follow-up time of 7 years, a total of 849 incident cases of type 2 diabetes were identified in the full cohort. Of these, 607 remained for analyses after applying similar exclusion criteria. In agreement with the case-cohort design, 56 individuals who developed type 2 diabetes during follow-up were identified in the sub-cohort (so called internal cases [24]), while 551 incident cases were identified outside the sub-cohort (external cases).

Clinical and biomarker measurements A total of $30 \mathrm{ml}$ of venous blood was collected at baseline from participants; it was fractionated into serum, plasma, buffy coat and erythrocytes and was liquated into straws and stored in liquid nitrogen at $170^{\circ} \mathrm{C}$ for conservation until the time of analysis. Serum and plasma samples were transferred to the Department of Internal Medicine, University of Tübingen, on dry ice for analyses. Serum ferritin concentrations were determined using an ADVIA Centaur XP and serum sTfR concentrations using a BN ProSpec System (both Siemens Healthcare, Erlangen, Germany). Plasma levels of alanine aminotransferase (ALT), $\gamma$-glutamyltransferase (GGT), HDL-cholesterol, triacylglycerol and high-sensitivity $\mathrm{C}$-reactive protein (hs-CRP) were determined using the automatic ADVIA 1650 analyser (Siemens Medical Solutions, Erlangen, Germany). Total adiponectin concentration was measured using an ELISA (Linco Research, St Charles, MI, USA). For determination of fetuin-A an immunoturbidimetric method was used with specific polyclonal goat anti-human fetuin-A antibodies to human fetuin-A (BioVendor Laboratory Medicine, Modreci,
Czech Republic) [25]. Erythrocyte levels of $\mathrm{HbA}_{1 \mathrm{c}}$ were measured using an HPLC procedure according to the manufacturer's instructions (Tosoh, Stuttgart, Germany). Total adiponectin concentration was measured using an ELISA (Linco Research). All assay procedures were performed as described by the manufacturer.

Statistical analysis The correlation between biomarkers of body iron stores and diabetes risk factors was studied in the sub-cohort with age- and sex-adjusted partial Pearson correlation coefficients. Skewed variables were logtransformed. Circulating levels of ferritin, sTfR and the ratio of STfR to ferritin were categorised into sex-specific quintiles based on sub-cohort participants. The RR of type 2 diabetes with $95 \%$ CI for quintiles of measures of body iron stores was calculated using the proportional hazards model, modified for the case-cohort design according to the Prentice method [24]. Age at baseline in 1-year categories was entered as a stratum variable. Age was also used as an underlying time variable in the counting processes, with entry defined as the participant's age at recruitment and exit defined as age at diabetes diagnosis or censoring. Models were adjusted for sex, BMI (continuous), waist circumference (continuous), education (in or no training, vocational training, technical school, or technical college or university degree), occupational activity (light, moderate or heavy), sports activity ( 0 , $0.1-4$ or over $4 \mathrm{~h} /$ week $)$, cycling $(0,0.1-2.4,2.5-4.9$ or 5 or more $\mathrm{h}$ /week), smoking habit (never, past, current $\leq 20$ cigarettes/day or current $>20$ cigarettes/day) and alcohol intake ( 0 , $0.1-5,5.1-10,10.1-20,20.1-40$ or over $40 \mathrm{~g}$ /day). In further models, we additionally adjusted for metabolic biomarkers (hs-CRP; GTT and ALT; HDL-cholesterol and triacylglycerol; and adiponectin, all continuous). In addition, a model including energy intake and consumption of coffee, red meat and whole-grain bread was used. In additional analyses we excluded individuals $(n=46)$ in whom serum ferritin was elevated beyond three times the SD from the mean (25 women with ferritin over $37.3 \mathrm{mg} / 1,21$ men with ferritin over $100.0 \mathrm{mg} / \mathrm{l}$ ) to reduce the possibility of including those with undiagnosed obvious haemochromatosis. Tests of linear trends in RR across quintiles of the biomarker levels were carried out by including the quintile of iron store variable in the model as a continuous variable. Since no evidence of a statistical interaction between measures of body iron and sex was observed in a model including an interaction term, the analyses were performed in men and women combined ( $p$ for interaction $0.80,0.24$ and 0.69 for ferritin, sTfR and the ratio of sTfR to ferritin, respectively).

All $p$ values presented are two-tailed, and $p<0.05$ was considered statistically significant. All analyses were performed using SAS software (version 9.1; SAS Institute Inc, Cary, NC, USA). 


\section{Results}

Baseline characteristics across quintiles of ferritin in the random sub-cohort are presented in Table 1. Participants with higher ferritin concentrations tended to be older, more inactive and have a higher BMI and waist circumference, and they reported higher alcohol consumption compared with participants who had lower ferritin concentrations. Consumption of red meat and coffee increased across quintiles of serum ferritin, while consumption of whole-grain bread decreased. Concentrations of the hepatic enzymes ALT and GGT, as well as hs-CRP and triacylglycerol, were higher with increasing ferritin categories, whereas adiponectin and HDL-cholesterol concentrations were lower.

The markers of iron stores were weakly to moderately correlated with other metabolic biomarkers and with BMI and waist circumference (Table 2). For example, the age- and sexadjusted partial Pearson coefficients for the correlations of waist circumference with ferritin, sTfR and sTfR-to-ferritin ratio were $0.23(p<0.001), 0.11(p<0.001)$ and $-0.18(p<0.001)$, respectively.

A significant association was observed between serum ferritin concentration and type 2 diabetes risk (Table 3 ). The relative risk in the highest compared with the lowest quintile of ferritin was 2.00 (95\% CI 1.35, 2.95; $\left.p_{\text {trend }}<0.001\right)$ when adjusted for age, sex, BMI, waist circumference, sports activity, bicycling, education, occupational activity, smoking habit and alcohol consumption. We next examined the impact of adjustment for different biomarkers on the association of measures of iron status with risk of type 2 diabetes. In these analyses, adjustment for GGT and ALT tended to have the strongest effect in terms of attenuating the risk for the association of ferritin with risk of diabetes, whereas adjustment for hs-CRP tended to have a weaker effect. Results remained virtually unchanged when hs-CRP was adjusted for in established risk categories $(<1,1-3, \geq 3 \mathrm{mg} / \mathrm{l})$ [26] instead of using a linear term. Although mutual adjustment for hs-CRP, GGT, ALT, adiponectin, HDL-cholesterol and triacylglycerol further attenuated the association, it still remained significant.

Table 1 Characteristics of study participants according to quintiles of plasma ferritin level in the sub-cohort

\begin{tabular}{|c|c|c|c|c|c|}
\hline \multirow[t]{2}{*}{ Characteristic } & \multicolumn{5}{|c|}{ Quintiles of plasma ferritin ${ }^{\mathrm{a}}$} \\
\hline & $1(n=377)$ & $2(n=394)$ & $3(n=389)$ & $4(n=398)$ & $5(n=411)$ \\
\hline Age (years), mean (SD) & $47.1(8.74)$ & $48.1(8.73)$ & $48.9(8.86)$ & $51.4(8.63)$ & $54.6(8.16)$ \\
\hline Sex $(\%$ men $)$ & 39.3 & 34.0 & 36.5 & 40.5 & 38.2 \\
\hline Current or former smoker $(\%)$ & 54.4 & 53.8 & 50.4 & 50.0 & 52.1 \\
\hline University degree education (\%) & 40.6 & 37.8 & 42.4 & 37.7 & 31.1 \\
\hline BMI $\left(\mathrm{kg} / \mathrm{m}^{2}\right)$, mean $(\mathrm{SD})$ & $24.7(3.59)$ & $25.0(4.09)$ & $26.1(3.97)$ & $26.2(4.15)$ & $27.8(4.51)$ \\
\hline Waist circumference $(\mathrm{cm})$, mean (SD) & $81.8(11.46)$ & $82.2(12.3)$ & $85.5(12.4)$ & $86.3(11.9)$ & $90.6(12.8)$ \\
\hline Alcohol consumption (g/day), mean (SD) & $13.1(16.18)$ & $11.2(12.5)$ & $13.6(19.9)$ & $15.1(17.9)$ & $17.0(29.1)$ \\
\hline Sports activity (h/week), mean (SD) & $1.33(2.26)$ & $1.05(1.64)$ & $1.04(1.76)$ & $1.03(1.79)$ & $0.69(1.29)$ \\
\hline Bicycling (h/week), mean (SD) & $1.87(2.73)$ & $1.76(2.89)$ & $1.48(2.22)$ & $1.99(2.88)$ & $1.86(3.06)$ \\
\hline Heavy or very heavy work strain $(\%)$ & 5.84 & 5.58 & 5.40 & 6.03 & 8.03 \\
\hline Energy intake (kJ/day), mean (SD) & $8,986(2847)$ & $8,642(2731)$ & $8,543(2506)$ & $8,647(2688)$ & $8,459(2874)$ \\
\hline Red meat (servings/day), mean (SD) & $0.26(0.20)$ & $0.28(0.19)$ & $0.28(0.19)$ & $0.29(0.18)$ & $0.30(0.20)$ \\
\hline Whole-grain bread (servings/day), mean (SD) & $0.96(1.03)$ & $0.96(1.05)$ & $0.97(1.14)$ & $0.94(1.15)$ & $0.77(0.98)$ \\
\hline Coffee (cups/day), mean (SD) & $2.78(2.39)$ & $2.74(1.82)$ & $2.75(2.12)$ & $2.86(2.15)$ & $2.81(2.00)$ \\
\hline \multicolumn{6}{|l|}{ Plasma levels, geometric mean $(95 \% \mathrm{CI})$} \\
\hline $\mathrm{sTfR}(\mathrm{mg} / \mathrm{l})$ & $1.09(1.06,1.12)$ & $1.00(0.98,1.02)$ & $0.98(0.96,1.00)$ & $1.00(0.98,1.02)$ & $1.02(1.00,1.04)$ \\
\hline $\mathrm{sTfR} /$ ferritin ratio & $0.58(0.53,0.64)$ & $0.23(0.22,0.25)$ & $0.13(0.12,0.14)$ & $0.08(0.08,0.09)$ & $0.04(0.04,0.05)$ \\
\hline Adiponectin (ng/ml) & $7.39(7.05,7.76)$ & $7.26(6.92,7.62)$ & $7.30(6.94,7.67)$ & $7.15(6.81,7.51)$ & $7.02(6.68,7.38)$ \\
\hline GGT (U/l) & $14.1(13.0,15.2)$ & $14.7(13.7,15.7)$ & $18.6(17.1,20.3)$ & $19.3(17.9,20.9)$ & $25.4(23.3,27.8)$ \\
\hline ALT (U/l) & $17.2(16.5,18.0)$ & $17.7(16.9,18.5)$ & $19.4(18.5,20.4)$ & $19.6(18.7,20.5)$ & $24.1(22.8,25.5)$ \\
\hline Fetuin-A $(\mu \mathrm{g} / \mathrm{ml})$ & $250(245,256)$ & $247(240,253)$ & $245(238,253)$ & $243(238,249)$ & $237(232,243)$ \\
\hline hs-CRP (mg/l) & $0.52(0.45,0.59)$ & $0.55(0.48,0.63)$ & $0.80(0.70,0.90)$ & $0.87(0.76,0.99)$ & $1.02(0.89,1.16)$ \\
\hline HDL-cholesterol (mmol/1) & $1.34(1.31,1.38)$ & $1.35(1.32,1.39)$ & $1.32(1.28,1.36)$ & $1.29(1.26,1.32)$ & $1.26(1.23,1.30)$ \\
\hline Triacylglycerol (mmol/l) & $1.07(1.01,1.13)$ & $1.02(0.97,1.08)$ & $1.17(1.11,1.23)$ & $1.26(1.19,1.34)$ & $1.40(1.32,1.48)$ \\
\hline
\end{tabular}

${ }^{a}$ Ferritin quintiles for men were $<8.00,8.00$ to $<13.0,13.0$ to $<19.0,19.0$ to $<28.0$ and $\geq 28.0 \mathrm{mg} / \mathrm{l}$; and for women ferritin quintiles were $<2.00$, 2.00 to $<3.89,3.89$ to $<6.39,6.39$ to $<11.0$, and $\geq 11.0 \mathrm{mg} / 1$ 
Table 2 Age and sex adjusted partial Pearson correlation coefficients between serum levels ${ }^{\mathrm{a}}$ of iron markers and biomarkers of selected risk factors of diabetes in the sub-cohort
No. of individuals included in sub-cohort, $n=1,969$

${ }^{a}$ Based on log-transformed values of biomarkers

\begin{tabular}{|c|c|c|c|c|c|c|}
\hline \multirow[b]{2}{*}{ Marker or biomarker } & \multicolumn{2}{|l|}{ Ferritin } & \multicolumn{2}{|l|}{ sTfR } & \multicolumn{2}{|c|}{ sTfR/ferritin ratio } \\
\hline & $r$ & $p$ value & $r$ & $p$ value & $r$ & $p$ value \\
\hline sTfR & -0.16 & $<0.001$ & & & & \\
\hline $\mathrm{sTfR} /$ ferritin ratio & -0.97 & $<0.001$ & 0.41 & $<0.001$ & & \\
\hline BMI & 0.21 & $<0.001$ & 0.10 & $<0.001$ & -0.17 & $<0.001$ \\
\hline Waist circumference & 0.23 & $<0.001$ & 0.11 & $<0.001$ & -0.18 & $<0.001$ \\
\hline Red meat intake & 0.09 & $<0.001$ & 0.01 & 0.51 & -0.08 & $<0.001$ \\
\hline Adiponectin & -0.08 & $<0.001$ & 0.02 & 0.32 & 0.08 & $<0.001$ \\
\hline GGT & 0.26 & $<0.001$ & 0.08 & $<0.001$ & -0.22 & $<0.001$ \\
\hline ALT & 0.24 & $<0.001$ & 0.07 & 0.003 & -0.21 & $<0.001$ \\
\hline Fetuin-A & -0.04 & 0.07 & 0.08 & $<0.001$ & 0.06 & 0.01 \\
\hline hs-CRP & 0.16 & $<0.001$ & 0.04 & 0.11 & -0.14 & $<0.001$ \\
\hline HDL-cholesterol & -0.08 & $<0.001$ & -0.03 & 0.14 & 0.07 & 0.003 \\
\hline Triacylglycerol & 0.13 & $<0.001$ & 0.03 & 0.20 & -0.15 & $<0.001$ \\
\hline
\end{tabular}

Specifically, the RR for the extreme quintile of ferritin was $1.60\left(95 \%\right.$ CI $\left.1.07,2.41 ; p_{\text {trend }}=0.007\right)$. In contrast to ferritin, no significant association was observed between serum levels of sTfR and type 2 diabetes risk. The corresponding multivariate RR for the highest vs lowest quintile of sTfR was 1.22 (95\% CI 0.81, 1.86; $\left.p_{\text {trend }}=0.64\right)$. Further adjustment for ferritin did not notably alter the result (data not shown). Finally, a significant inverse association was observed between sTfR-to-ferritin ratio and type 2 diabetes risk in models adjusting for various lifestyle factors (RR 0.50; 95\% CI $\left.0.34,0.73 ; p_{\text {trend }}<0.001\right)$. Only marginal changes in risk estimates were observed in models further adjusting for different biomarkers. In a final model including markers of inflammation and dyslipidaemia, as well as adiponectin and liver enzymes, the RR for extreme quintiles of sTfR-toferritin ratio was 0.61 (95\% CI $\left.0.41,0.91 ; p_{\text {trend }}=0.02\right)$. Further adjustment for dietary factors, including energy intake, consumption of red and processed meat, coffee, whole-grain bread, magnesium and dietary iron did not notably alter the associations observed for ferritin and sTfR-to-ferritin ratio (data not shown); neither did further adjustment for circulating $\mathrm{HbA}_{1 \mathrm{c}}$ level (data not shown).

When we excluded 46 individuals with serum ferritin levels greater than or equal to three times the SD from the mean, no notable alteration in the results was observed (data not shown). Results were also not substantially different when we excluded 157 cases of type 2 diabetes that occurred within the first 2 years of follow-up (to further reduce the possibility of including individuals with undiagnosed diabetes at baseline) (data not shown). In further exploratory analyses we investigated potential effect modification of $\mathrm{HbA}_{1 \mathrm{c}}$ level, BMI, hs-CRP level or hypertension. We found a significant interaction for circulating $\mathrm{HbA}_{1 \mathrm{c}}$ level on the association between ferritin and diabetes risk $\left(p_{\text {interaction }}<0.001\right)$. Thus, the association observed between serum ferritin level and diabetes risk was significant among persons with $\mathrm{HbA}_{1 \mathrm{c}}$ of $5.3 \%(34.4 \mathrm{mmol} / \mathrm{mol})$ or more (RR for the highest quintile $1.70 ; 95 \%$ CI 1.03 , 2.80 ), but not among persons with $\mathrm{HbA}_{1 \mathrm{c}}$ values below $5.3 \%(34.4 \mathrm{mmol} / \mathrm{mol})$ (RR for the highest quintile 0.97 ; CI $0.38,2.50)$. Circulating $\mathrm{HbA}_{1 \mathrm{c}}$ also tended to modify the association between STfR and diabetes risk, albeit the interaction term did not reach the significance level of 0.05 $\left(p_{\text {interaction }}=0.08\right)$. Thus, among participants with an $\mathrm{HbA}_{1 \mathrm{c}}$ level above 5.3\% (34.4 $\left.\mathrm{mmol} / \mathrm{mol}\right)$, the RR for the highest vs lowest quintile was 1.15 (95\% CI 0.69 , 1.91), whereas for $\mathrm{HbA}_{1 \mathrm{c}}$ levels below 5.3\% (34.4 mmol/ mol) it was $2.39(95 \%$ CI $0.79,7.23)$. We found no significant interaction of ferritin, sTfR and the sTfR-to-ferritin ratio with BMI, hs-CRP level or hypertension, on risk of type 2 diabetes (data not shown).

\section{Discussion}

In the present case-cohort study, higher body iron stores (reflected by elevated ferritin concentration and a lower ratio of sTfR to ferritin) were associated with increased risk of type 2 diabetes. These associations remained significant after adjustment for a wide range of known risk factors and markers of different pathways of the development of diabetes. Of the individual markers, adjustment for ALT and GGT had the strongest impact on the association observed between ferritin and diabetes risk. In contrast, only a marginal impact on the inverse association between sTfR-to-ferritin ratio and type 2 diabetes was observed in models adjusting for biomarkers of inflammation, hepatic fat accumulation, insulin resistance and dyslipidaemia. Levels of sTfR were not significantly associated with risk of type 2 diabetes. 
Table 3 RR of type 2 diabetes $(95 \% \mathrm{CI})$ according to serum ferritin, sTfR and the ratio of sTfR to ferritin

\begin{tabular}{|c|c|c|c|c|c|c|}
\hline \multirow[t]{2}{*}{ Risk factor } & \multicolumn{5}{|c|}{$\mathrm{RR}(95 \% \mathrm{CI})$} & \multirow[t]{2}{*}{$p_{\text {trend }}$} \\
\hline & Quintile 1 & Quintile 2 & Quintile 3 & Quintile 4 & Quintile 5 & \\
\hline \multicolumn{7}{|l|}{ Ferritin } \\
\hline Men, mg/l & $<8.00$ & 8.00 to $<13.0$ & 13.0 to $<19.0$ & 19.0 to $<28.0$ & $\geq 28.0$ & \\
\hline Women, mg/1 & $<2.00$ & 2.00 to $<3.89$ & 3.89 to $<6.39$ & 6.39 to $<11.0$ & $\geq 11.0$ & \\
\hline Model 1 & 1 & $0.95(0.59,1.50)$ & $1.49(1.00,2.24)$ & $1.54(1.03,2.31)$ & $2.00(1.35,2.95)$ & $<0.001$ \\
\hline Model $1+$ hs-CRP & 1 & $1.01(0.63,1.63)$ & $1.56(1.03,2.37)$ & $1.65(1.09,2.50)$ & $2.09(1.40,3.12)$ & $<0.001$ \\
\hline Model $1+$ GGT and ALT & 1 & $0.91(0.57,1.46)$ & $1.45(0.97,2.17)$ & $1.51(1.01,2.26)$ & $1.74(1.17,2.60)$ & $<0.001$ \\
\hline Model $1+$ fetuin-A & 1 & $0.94(0.59,1.51)$ & $1.53(1.01,2.30)$ & $1.58(1.05,2.38)$ & $2.13(1.43,3.16)$ & $<0.001$ \\
\hline Model $1+$ adiponectin & 1 & $0.92(0.57,1.49)$ & $1.52(1.00,2.30)$ & $1.55(1.03,2.33)$ & $1.95(1.31,2.90)$ & $<0.001$ \\
\hline Model $1+$ HDL-cholesterol, triacylglycerol & 1 & $1.00(0.62,1.61)$ & $1.49(0.99,2.25)$ & $1.49(0.99,2.23)$ & $1.80(1.22,2.66)$ & $<0.001$ \\
\hline Model 2 & 1 & $1.00(0.61,1.65)$ & $1.49(0.97,2.30)$ & $1.59(1.05,2.42)$ & $1.73(1.15,2.61)$ & 0.002 \\
\hline \multicolumn{7}{|l|}{ sTfR } \\
\hline Men, mg/l & $<0.90$ & 0.90 to $<1.00$ & 1.00 to $<1.10$ & 1.10 to $<1.30$ & $\geq 1.30$ & \\
\hline Women, mg/1 & $<0.80$ & 0.80 to $<0.89$ & 0.89 to $<1.00$ & 1.00 to $<1.20$ & $\geq 1.20$ & \\
\hline Model 1 & 1 & $1.37(0.89,2.12)$ & $1.83(1.20,2.79)$ & $1.52(1.03,2.24)$ & $1.22(0.81,1.86)$ & 0.64 \\
\hline Model $1+$ hs-CRP & 1 & $1.42(0.92,2.19)$ & $1.87(1.23,2.85)$ & $1.51(1.02,2.23)$ & $1.18(0.78,1.80)$ & 0.85 \\
\hline Model $1+$ GGT and ALT & 1 & $1.33(0.86,2.07)$ & $1.81(1.19,2.76)$ & $1.46(0.99,2.16)$ & $1.19(0.78,1.81)$ & 0.75 \\
\hline Model $1+$ fetuin-A & 1 & $1.36(0.88,2.10)$ & $1.78(1.17,2.71)$ & $1.48(1.00,2.18)$ & $1.14(0.75,1.73)$ & 0.94 \\
\hline Model $1+$ adiponectin & 1 & $1.40(0.89,2.20)$ & $1.81(1.17,2.81)$ & $1.63(1.09,2.45)$ & $1.30(0.84,2.00)$ & 0.38 \\
\hline Model $1+$ HDL-cholesterol, triacylglycerol & 1 & $1.47(0.94,2.30)$ & $1.93(1.25,2.97)$ & $1.50(1.00,2.23)$ & $1.36(0.89,2.08)$ & 0.47 \\
\hline Model 2 & 1 & $1.38(0.86,2.20)$ & $1.87(1.20,2.93)$ & $1.47(0.97,2.22)$ & $1.21(0.78,1.89)$ & 0.80 \\
\hline \multicolumn{7}{|l|}{ sTfR-to-ferritin ratio } \\
\hline Men, $(\mathrm{mg} / \mathrm{l}) /(\mathrm{mg} / \mathrm{l})$ & $<0.04$ & 0.04 to $<0.05$ & 0.05 to $<0.08$ & 0.08 to $<0.13$ & $\geq 0.13$ & \\
\hline Women, $(\mathrm{mg} / \mathrm{l}) /(\mathrm{mg} / \mathrm{l})$ & $<0.09$ & 0.09 to $<0.15$ & 0.15 to $<0.25$ & 0.25 to $<0.50$ & $\geq 0.50$ & \\
\hline Model 1 & 1 & $0.66(0.48,0.90)$ & $0.59(0.43,0.82)$ & $0.63(0.45,0.88)$ & $0.50(0.34,0.73)$ & $<0.001$ \\
\hline Model $1+$ hs-CRP & 1 & $0.67(0.49,0.91)$ & $0.59(0.42,0.82)$ & $0.63(0.45,0.89)$ & $0.47(0.32,0.70)$ & $<0.001$ \\
\hline Model $1+$ GGT and ALT & 1 & $0.72(0.52,0.99)$ & $0.66(0.47,0.91)$ & $0.69(0.49,0.98)$ & $0.56(0.38,0.83)$ & 0.004 \\
\hline Model $1+$ fetuin-A & 1 & $0.64(0.46,0.87)$ & $0.57(0.41,0.79)$ & $0.58(0.41,0.82)$ & $0.46(0.32,0.68)$ & $<0.001$ \\
\hline Model $1+$ adiponectin & 1 & $0.71(0.51,0.97)$ & $0.62(0.44,0.87)$ & $0.64(0.45,0.92)$ & $0.52(0.35,0.76)$ & $<0.001$ \\
\hline Model $1+$ HDL-cholesterol, triacylglycerol & 1 & $0.69(0.50,0.95)$ & $0.64(0.46,0.89)$ & $0.71(0.50,1.00)$ & $0.55(0.37,0.80)$ & 0.002 \\
\hline Model 2 & 1 & $0.76(0.54,1.06)$ & $0.67(0.47,0.96)$ & $0.70(0.48,1.02)$ & $0.57(0.38,0.85)$ & 0.005 \\
\hline
\end{tabular}

Model 1 is adjusted for age, sex, BMI, waist circumference, sports activity, bicycling, education, occupational activity, smoking habit and alcohol consumption

Model 2 is adjusted for factors in model 1 and GGT, ALT, fetuin-A, hs-CRP, adiponectin, HDL-cholesterol and triacylglycerol concentrations

The results of the present study are consistent with findings from previous prospective studies suggesting that high serum ferritin concentrations $[4-6,13]$ and lower ratios of sTfR to ferritin $[5,7]$ are associated with increased risk of type 2 diabetes. Specifically, in a prospective study among Finnish men, Salonen et al [7] observed a 2.5-fold higher risk of diabetes among persons in the lowest compared with the highest quintile of sTfR-to-ferritin ratio; however, their analysis was not adjusted for markers of inflammation, liver enzymes or adiponectin. In the Nurses' Health Study [5], serum ferritin levels and the sTfR-to-ferritin ratio were associated with an approximately 2.5 -fold higher diabetes risk in the highest vs lowest quintile after controlling for C-reactive protein and various conventional risk factors of diabetes. In the EPIC-Norfolk cohort [4], higher ferritin level was associated with diabetes risk after adjustment for conventional risk factors as well as plasma levels of vitamin C, C-reactive protein, fibrinogen, IL-6, liver function tests, ALT, GGT and adiponectin. In contrast to these studies, in the ARIC cohort the association was not significant after adjustment for HDLcholesterol, waist circumference, hypertension, fasting glucose level and fasting triacylglycerol level [13]. In the present study, we were able to investigate the association of body iron stores, as assessed not only as circulating ferritin concentrations but also as sTfR (and the ratio of sTfR to ferritin), with risk of incident type 2 diabetes in a population-based cohort. 
In addition, we were able to account for potential confounding effects of a wide range of biomarkers presenting different pathways leading to onset of type 2 diabetes (including hsCRP, adiponectin, GGT, ALT, fetuin-A, HDL-cholesterol and triacylglycerol), some of which were not accounted for in previous studies. Generally, risk estimates between measures of body iron status and type 2 diabetes were attenuated after inclusion of biomarkers. Exceptions were C-reactive protein and fetuin-A, for which risk estimates increased; however, the increase was small and lay within the limits of uncertainty. We were also able to adjust for circulating $\mathrm{HbA}_{1 \mathrm{c}}$, which, however, as a measure of long-term blood glucose levels, can be considered a rather downstream marker of onset of disease rather than a metabolic pathway to type 2 diabetes. Therefore, we excluded $\mathrm{HbA}_{1 \mathrm{c}}$ levels from the main analysis. Interestingly, though, the relative contributions of the biomarkers were not substantially different when we further adjusted for $\mathrm{HbA}_{1 \mathrm{c}}$ levels in sensitivity analyses.

Two prospective studies that evaluated the association between ferritin and diabetes incidence also measured sTfR levels and reported that not only a high ferritin level but also a low sTfR-to-ferritin ratio was significantly associated with an increased risk of diabetes [5, 7]. However, the independent association between sTfR levels per se and diabetes risk was reported only in one study. That study, conducted within the placebo arm of the Diabetes Prevention Program, suggested that elevated sTfR levels are associated with increased risk of diabetes among overweight and obese persons with impaired glucose tolerance [6]. The authors speculated that sTfR levels may increase as a compensatory mechanism for a reduction in free iron levels that may occur secondary to oxidative stress. Alternatively, the authors suggested that STfR may be a biomarker of some other factor that is causally related to development of diabetes, possibly unrelated to iron load. In the present study, we found no association between sTfR and diabetes incidence. Interestingly, sTfR tended to be associated with diabetes risk in individuals with $\mathrm{HbA}_{1 \mathrm{c}}$ values below median but not in those with above-median $\mathrm{HbA}_{1 \mathrm{c}}$ values. In the present study circulating levels of sTfR were remarkably lower (average $1.13 \mathrm{mg} / \mathrm{l}$ ) than those observed in the Diabetes Prevention Program (average $3.4 \mathrm{mg} / \mathrm{l}$ ). Thus, it is possible that in the present study the contrast between low and high values was too small to reveal an association with increased risk.

The mechanism for the association between ferritin and type 2 diabetes is unknown, but excess iron is usually stored in the liver, muscle and pancreas and may cause organspecific oxidative stress leading to insulin resistance and beta cell failure. Iron is a catalyst in the formation of hydroxyl radicals, which are highly active and potentially attack cell membrane lipids [27], and subsequent changes in cell membrane may lead to impaired glucose intake of the cell $[28,29]$. In the liver, damage due to oxidative stress may cause insulin resistance by interfering with the ability of insulin to suppress hepatic glucose production [30]. The hepatic insulin resistance caused by iron overload seems to be independent of insulin resistance induced by fatty liver disease [31]. In addition, iron accumulation in hepatocytes may interfere with the insulin-extracting capacity of the liver, while iron accumulation also interferes with insulin synthesis and secretion in the pancreas [2]. Hence, iron excess seems to contribute initially to insulin resistance and subsequently to decreased insulin secretion [2]. In addition, iron deposition in muscle has been shown to decrease glucose uptake [32]. Although elevated plasma ferritin levels may be causally related to risk of diabetes, increased ferritin levels may also be a marker of metabolic alterations that may causally increase diabetes risk. For example, increased ferritin values may reflect subclinical systemic inflammation [1, 33], fatty liver [34, 35] or dyslipidaemia [11, 12], which are all potentially related to insulin resistance and risk of type 2 diabetes. However, in the present study the association observed between ferritin and risk of diabetes was independent of biomarkers of inflammation, hepatic fat accumulation, insulin resistance and dyslipidaemia.

Circulating ferritin concentrations are commonly used as an indicator of body iron stores in epidemiological studies. This use, however, is complicated, because ferritin is an acute-phase protein that may be elevated in inflammation, severe liver disease or cancer [8]. Furthermore, its relationship to nontransferring bound iron and the intracellular iron pool, which may be responsible for iron-related oxidative damage, is uncertain [14]. Circulating sTfR concentration has been proposed as a marker of iron status that is less affected by the presence of inflammation. However, low iron stores result in the induction of synthesis of TfRs and they also reflect iron requirement [36]. Therefore, it has been suggested that the ratio of serum concentrations of sTfR and ferritin should be a preferred measure of body iron stores [18, 19, 37]. However, in the present study no notable difference in the strength of the associations with diabetes risk was observed between ferritin and sTfR-to-ferritin ratio. Given that a strong inverse correlation $(r=-0.97)$ was observed between ferritin and sTfR-to-ferritin ratio, and no association was observed for sTfR, the association observed for sTfR-to-ferritin ratio is likely to be driven by ferritin only. On the other hand it can be speculated that if sTfR was a more accurate marker of iron stores, then this would indicate that iron stores are not a relevant risk factor for the development of diabetes and that ferritin concentration might reflect something different not fully accounted for in the present-as well as in previous-studies showing a strong association between ferritin and diabetes risk.

All potential cases in our study were verified by a physician. Although we considered only clinically apparent type 2 diabetes and did not screen our study population during 
follow-up, we excluded participants with plasma glucose values within the diabetic range at baseline, and exclusion of individuals diagnosed within the first 2 years of follow-up did not alter the result. Thus, it is unlikely that prevalent but undiagnosed cases of diabetes have influenced the results. A number of participants were excluded from the original study population for the present analysis. However, excluded cases and non-cases did not differ from those cases and non-cases included in the analysis and we therefore do not expect an influence of exclusion on the risk estimates. We had data available from a single blood withdrawal only, which might have introduced random measurement errors in determining biomarkers. Random measurement error generally tends to bias relative risks in epidemiological studies between biomarkers and disease outcomes towards the null [38]. Also, additional sources of measurement variability (related to blood processing or long-term storage) may have differed between the biomarkers. Thus, we cannot entirely rule out the possibility that some of the above-mentioned limitations may have influenced our results. Although the analyses were adjusted for a large variety of known diabetes risk factors, we cannot rule out the possibility that our observations may have been influenced by other unmeasured factors or by residual confounding due to imprecision in the measurement of covariates.

In conclusion, we found that elevated body iron stores below the levels observed in haemochromatosis are associated with higher risk of type 2 diabetes independently of established risk factors and a range of diabetes biomarkers. However, sTfR levels were not associated with type 2 diabetes and our findings imply that particularly high ferritin levels are related to a higher risk of type 2 diabetes. Further research is warranted to identify predictors of ferritin concentrations, which may help to identify targets for lowering the risk of type 2 diabetes.

\begin{abstract}
Acknowledgements This study was partly supported by grants from the Federal Ministry of Education and Research, Germany (Bundesministerium für Bildung und Forschung, Förderkennzeichen 0315381A and B). The funding agency had no role in study design, data collection and analysis, decision to publish or preparation of the manuscript. The responsibility for the content of this manuscript lies with the authors.
\end{abstract}

Duality of interest The authors declare that there is no duality of interest associated with this manuscript.

Contribution statement All authors contributed to the study conception and design, analysis and interpretation of data and critically revised and approved the final version of the article to be published.

Open Access This article is distributed under the terms of the Creative Commons Attribution License which permits any use, distribution, and reproduction in any medium, provided the original author(s) and the source are credited.

\section{References}

1. Witte DL, Crosby WH, Edwards CQ, Fairbanks VF, Mitros FA (1996) Practice guideline development task force of the College of American Pathologists. Hereditary hemochromatosis. Clin Chim Acta 245:139-200

2. Wilson JG, Lindquist JH, Grambow SC, Crook ED, Maher JF (2003) Potential role of increased iron stores in diabetes. Am J Med Sci 325:332-339

3. Eshed I, Elis A, Lishner M (2001) Plasma ferritin and type 2 diabetes mellitus: a critical review. Endocr Res 27:91-97

4. Forouhi NG, Harding AH, Allison M et al (2007) Elevated serum ferritin levels predict new-onset type 2 diabetes: results from the EPIC-Norfolk prospective study. Diabetologia 50:949-956

5. Jiang R, Manson JE, Meigs JB, Ma J, Rifai N, Hu FB (2004) Body iron stores in relation to risk of type 2 diabetes in apparently healthy women. JAMA 291:711-717

6. Rajpathak SN, Wylie-Rosett J, Gunter MJ et al (2009) Biomarkers of body iron stores and risk of developing type 2 diabetes. Diabetes Obes Metab 11:472-479

7. Salonen JT, Tuomainen TP, Nyyssonen K, Lakka HM, Punnonen K (1998) Relation between iron stores and non-insulin dependent diabetes in men: case-control study. BMJ 317:727

8. Ponka P, Beaumont C, Richardson DR (1998) Function and regulation of transferrin and ferritin. Semin Hematol 35:35-54

9. Lee C, Adler A, Sandhu M et al (2009) Association of C-reactive protein with type 2 diabetes: prospective analysis and metaanalysis. Diabetologia 52:1040-1047

10. Choi KM, Lee KW, Kim HY et al (2005) Association among serum ferritin, alanine aminotransferase levels, and metabolic syndrome in Korean postmenopausal women. Metabolism 54:1510 1514

11. Halle M, Konig D, Berg A, Keul J, Baumstark MW (1997) Relationship of serum ferritin concentrations with metabolic cardiovascular risk factors in men without evidence for coronary artery disease. Atherosclerosis 128:235-240

12. Ramakrishnan U, Kuklina E, Stein AD (2002) Iron stores and cardiovascular disease risk factors in women of reproductive age in the United States. Am J Clin Nutr 76:1256-1260

13. Jehn ML, Guallar E, Clark JM et al (2007) A prospective study of plasma ferritin level and incident diabetes: the Atherosclerosis Risk in Communities (ARIC) Study. Am J Epidemiol 165:10471054

14. Lee DH, Jacobs DR Jr (2004) Serum markers of stored body iron are not appropriate markers of health effects of iron: a focus on serum ferritin. Med Hypotheses 62:442-445

15. Kohgo Y, Nishisato T, Kondo H, Tsushima N, Niitsu Y, Urushizaki I (1986) Circulating transferrin receptor in human serum. Br J Haematol 64:277-281

16. Ponka P, Lok CN (1999) The transferrin receptor: role in health and disease. Int J Biochem Cell Biol 31:1111-1137

17. Sherwood RA, Pippard MJ, Peters TJ (1998) Iron homeostasis and the assessment of iron status. Ann Clin Biochem 35(Pt 6):693-708

18. Punnonen K, Irjala K, Rajamaki A (1997) Serum transferrin receptor and its ratio to serum ferritin in the diagnosis of iron deficiency. Blood 89:1052-1057

19. Skikne BS, Flowers CH, Cook JD (1990) Serum transferrin receptor: a quantitative measure of tissue iron deficiency. Blood 75:1870-1876

20. Boeing H, Korfmann A, Bergmann MM (1999) Recruitment procedures of EPIC-Germany. European Investigation into Cancer and Nutrition. Ann Nutr Metab 43:205-215

21. Riboli E, Hunt KJ, Slimani N et al (2002) European Prospective Investigation into Cancer and Nutrition (EPIC): study populations and data collection. Public Health Nutr 5:1113-1124 
22. Boeing H, Wahrendorf J, Becker N (1999) EPIC-Germany-a source for studies into diet and risk of chronic diseases. European Investigation into Cancer and Nutrition. Ann Nutr Metab 43:195204

23. Klipstein-Grobusch K, Georg T, Boeing H (1997) Interviewer variability in anthropometric measurements and estimates of body composition. Int J Epidemiol 26(Suppl 1):S174-S180

24. Barlow WE, Ichikawa L, Rosner D, Izumi S (1999) Analysis of case-cohort designs. J Clin Epidemiol 52:1165-1172

25. Stefan N, Fritsche A, Weikert C et al (2008) Plasma fetuin-A levels and the risk of type 2 diabetes. Diabetes 57:2762-2767

26. Pearson TA, Mensah GA, Alexander RW et al (2003) Markers of inflammation and cardiovascular disease: application to clinical and public health practice: a statement for healthcare professionals from the Centers for Disease Control and Prevention and the American Heart Association. Circulation 107:499-511

27. Oberley LW (1988) Free radicals and diabetes. Free Radic Biol Med 5:113-124

28. Opara EC (2004) Role of oxidative stress in the etiology of type 2 diabetes and the effect of antioxidant supplementation on glycemic control. J Investig Med 52:19-23

29. Wolff SP (1993) Diabetes mellitus and free radicals. Free radicals, transition metals and oxidative stress in the aetiology of diabetes mellitus and complications. Br Med Bull 49:642-652
30. Fernandez-Real JM, Lopez-Bermejo A, Ricart W (2002) Cross-talk between iron metabolism and diabetes. Diabetes 51:2348-2354

31. Haap M, Machann J, von Friedeburg C et al (2011) Insulin sensitivity and liver fat: role of iron load. J Clin Endocrinol Metab 96:E958-E961

32. Merkel PA, Simonson DC, Amiel SA et al (1988) Insulin resistance and hyperinsulinemia in patients with thalassemia major treated by hypertransfusion. N Engl J Med 318:809-814

33. Hernandez C, Lecube A, Carrera A, Simo R (2005) Soluble transferrin receptors and ferritin in type 2 diabetic patients. Diabet Med 22:97-101

34. Hsiao TJ, Chen JC, Wang JD (2004) Insulin resistance and ferritin as major determinants of nonalcoholic fatty liver disease in apparently healthy obese patients. Int J Obes Relat Metab Disord 28:167-172

35. Mendler MH, Turlin B, Moirand R et al (1999) Insulin resistanceassociated hepatic iron overload. Gastroenterology 117:1155-1163

36. Chan LN, Gerhardt EM (1992) Transferrin receptor gene is hyperexpressed and transcriptionally regulated in differentiating erythroid cells. J Biol Chem 267:8254-8259

37. Malope BI, MacPhail AP, Alberts M, Hiss DC (2001) The ratio of serum transferrin receptor and serum ferritin in the diagnosis of iron status. Br J Haematol 115:84-89

38. Fleiss J (1986) Reliability of measurement. In: The design and analysis of clinical experiments, 1st edn. Wiley, New York, pp 1-32 